\title{
Analysis of Optical Neural Stimulation effects on neural networks affected by neurodegenerative diseases
}

\author{
M. Zverev, F. Fanjul-Vélez*, I Salas-García, N. Ortega-Quijano, J. L. Arce-Diego* \\ Applied Optical Techniques Group, TEISA Department, University of Cantabria, Av de los Castros \\ s/n, 39005, Santander (Spain)
}

\begin{abstract}
The number of people in risk of developing a neurodegenerative disease increases as the life expectancy grows due to medical advances. Multiple techniques have been developed to improve patient's condition, from pharmacological to invasive electrodes approaches, but no definite cure has yet been discovered.

In this work Optical Neural Stimulation (ONS) has been studied. ONS stimulates noninvasively the outer regions of the brain, mainly the neocortex. The relationship between the stimulation parameters and the therapeutic response is not totally clear. In order to find optimal ONS parameters to treat a particular neurodegenerative disease, mathematical modeling is necessary. Neural networks models have been employed to study the neural spiking activity change induced by ONS. Healthy and pathological neocortical networks have been considered to study the required stimulation to restore the normal activity. The network consisted of a group of interconnected neurons, which were assigned 2D spatial coordinates. The optical stimulation spatial profile was assumed to be Gaussian. The stimulation effects were modeled as synaptic current increases in the affected neurons, proportional to the stimulation fluence. Pathological networks were defined as the healthy ones with some neurons being inactivated, which presented no synaptic conductance. Neurons' electrical activity was also studied in the frequency domain, focusing specially on the changes of the spectral bands corresponding to brain waves. The complete model could be used to determine the optimal ONS parameters in order to achieve the specific neural spiking patterns or the required local neural activity increase to treat particular neurodegenerative pathologies.
\end{abstract}

Keywords: Optical Neural Stimulation, Neural network. Neurodegenerative diseases, Neural spiking

\section{INTRODUCTION}

$18.2 \%$ of European population is aged or over 65 in 2013 according to Eurostat [1]. By 2080 this percentage will be $28.7 \%$. As a consequence more people will suffer from pathologies in nervous system. Nowadays several techniques of nervous system stimulation have been studied. However, the definite cure has not been discovered yet. Noninvasive techniques, like transcranial magnetic stimulation (TMS) or transcranial direct current stimulation (tDCS), have been proven to be secure and efficient [2].

Outer deep brain regions can be stimulated noninvasively by optical radiation. The stimulation of deeper regions requires a surgical intervention [2]. Neural activity can be varied by optical radiation by employing ONS over specific brain regions. In this work ONS has been studied. ONS stimulates noninvasively the outer regions of the brain, mainly the neocortex. In order to find optimal ONS parameters to treat a particular neurodegenerative disease, mathematical modeling is necessary. Neural networks models have been employed to study the neural spiking activity change induced by ONS. Healthy and pathological neocortical networks will be considered to study the required stimulation to restore the normal activity. The network consists of a group of interconnected neurons, which were assigned 2D spatial coordinates. The optical stimulation spatial profile was assumed to be Gaussian. The stimulation effects were modeled as synaptic current increases in the affected neurons, proportional to the stimulation fluence. Pathological networks were defined as the healthy ones with some neurons being inactivated, which presented no synaptic conductance. Neurons' electrical activity was also studied in the frequency domain, focusing specially on the changes of the spectral bands corresponding to brain waves. The complete model could be used to determine the optimal ONS to treat particular neurodegenerative pathologies.

*fanjulf@unican.es; arcedj@unican.es; phone+34 94220 67 30; fax +34 9422018 73; www.teisa.unican.es/toa

Clinical and Translational Neurophotonics; Neural Imaging and Sensing; and Optogenetics and

Optical Manipulation, edited by Steen J. Madsen, et. al., Proc. of SPIE Vol. 9690, 96901Q

(C) 2016 SPIE · CCC code: 1605-7422/16/\$18 · doi: 10.1117/12.2208683

Proc. of SPIE Vol. $969096901 Q-1$ 
Section 2 contains the fundamentals of the optical propagation model of ONS. From this data in section 3 neural network model is described, as long as its relationship with optical radiation pattern. The main results of the work and presented and discussed in Section 4. Section 5 contains the final conclusions.

\section{LIGHT PROPAGATION}

One of the most usual ways to treat light propagation is by means of Monte Carlo methods [3]. In this work, according to the specifications of the application, open-source Mesh-based Monte Carlo method was used [6]. In this method packets of photons are launched to a head structure. Afterwards their trajectory and energy is observed. Multiple scattering events occur during the propagation. The energy of the photon package is attenuated between the scattering events as:

$$
\Delta W=\frac{\mu_{a}}{\mu_{e}} W
$$

where $W$ is the package weight (or energy), $\mu_{e}$ is the extinction coefficient and $\mu_{e}$ is the absorption coefficient of the medium. The polar angle of the scattered photon package is:

$$
\theta= \begin{cases}\operatorname{acos}\left\{\frac{1}{2 g}\left[1+g^{2}-\left(\frac{1-g^{2}}{1-g+2 g \xi_{2}}\right)^{2}\right]\right\}, & g \neq 0, \\ \operatorname{acos}\left(2 \xi_{2}-1\right) & , g=0\end{cases}
$$

being $g$ the scattering anisotropy. The $\xi_{2}$ is a pseudorandom number that defines the scattering events and their location, with uniform distribution between 0 and 1 . To ensure the accuracy of the results, the process of launching and propagating packages of photons is repeated $10^{6}$ times. Some results are shown in Figure 1. It can be appreciated that optical illumination shows a high degree of spatial specificity. Optical radiation is actually able to reach the cerebral cortex.

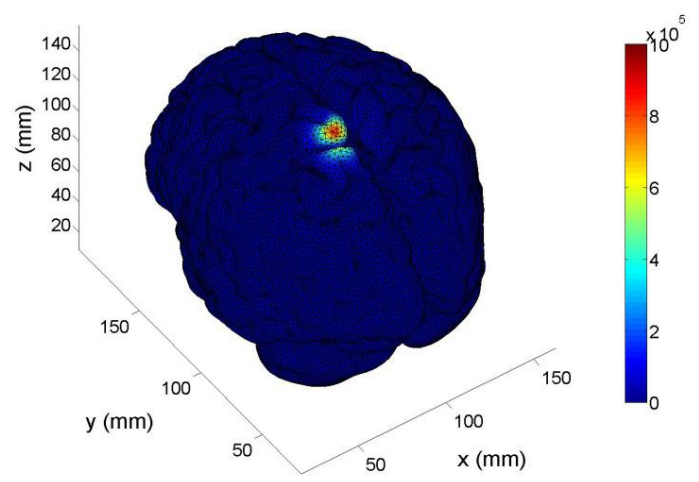

Figure 1. Optical propagation of a CW HeNe laser at $633 \mathrm{~nm}$ on the cerebral cortex.

\section{MODEL OF NEURAL NETWORKS AND STIMULATION}

ONS is able to stimulate a small region of neocortex. Neocortex is composed of a neural network that can be mathematically described. The most known neuron model is the one by Hodgkin and Huxley. This model can represent any neuron dynamic as it describes the action potential generation from the transmembrane ionic currents and membrane voltage [7]. In 2003 Izhikevich presented his simpler neuron model [8]. The model properly mimics neuronal behavior without taking into account the transmembrane ionic currents. As a consquence the Hodgkin and Huxley model is appropriate to study different effects on a single neuron, but the Izhikevich model will be more appropriate for networks, as it requires less calculus. Izhikevich model is described by the following equations system 


$$
\begin{aligned}
& v^{\prime}=0.04 v^{2}+5 v+140-u+I \\
& u^{\prime}=a(b v-u) \text {, } \\
& \text { if } \quad v \geq 30 \mathrm{mV} \\
& \text { then }\left\{\begin{array}{c}
v \leftarrow c \\
u \leftarrow u+d
\end{array}\right.
\end{aligned}
$$

where $v$ is the membrane voltage of the neuron, $u$ is the membrane recovery variable, $a, b, c$ and $d$ are constant parameters of the model and $I$ is the input current.

The ONS effect is directly related to temperature increase induced by optical radiation absorption in the target brain region [9]. As the tissue absorbs energy, its temperature grows and stimulated neurons' membranes are depolarized. Therefore the depolarization can be assumed proportional to the absorbed energy. Stimulation-induced depolarization is modeled as a membrane voltage increase in the stimulated neurons. This increase is proportional to the Gaussian function of the distance between the stimulated neuron and the central point of the stimulus incidence. Different depolarization voltages have been tested to adjust the Gaussian stimulus. Neurophysiological parameters, such as the axon's length and width, were modeled as white noise.

\section{RESULTS AND DISCUSSION}

In the particular case we are dealing with, and according to biological data, the considered neural network is located in a $100 \mu \mathrm{m} \times 100 \mu \mathrm{m}$ neocortical slab. As in [8], the network receives thalamic input, the number of inhibitory neurons is $1 / 5$ of the total and the rest of the neurons are excitatory. All the neurons are interconnected, and there is no connection of neurons to themselves. As in neurodegenerative diseases the neurons suffer different kinds of defects and end up dying, the pathology modeled in this study randomly inactivates or kills $60 \%$ of neurons. In this study the maximum depolarization is set to $50 \mathrm{mV}$ and the Gaussian function's variance to $100 \mu \mathrm{m}^{2}$.

a)

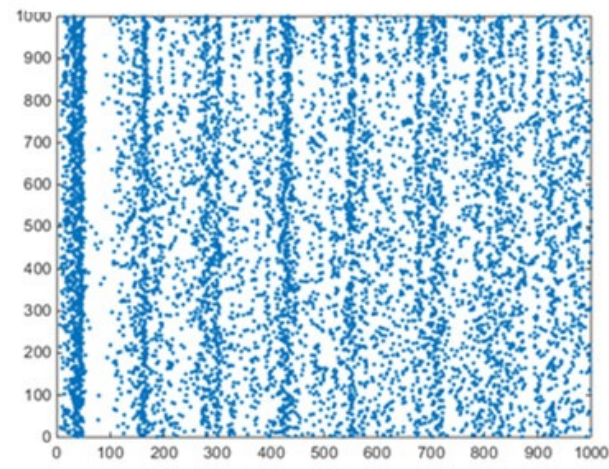

b)

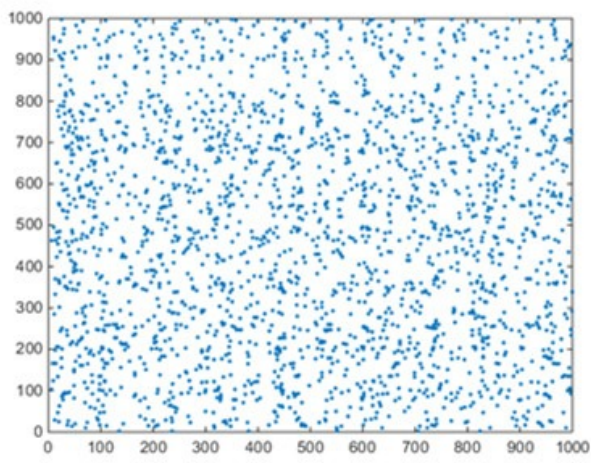

c)

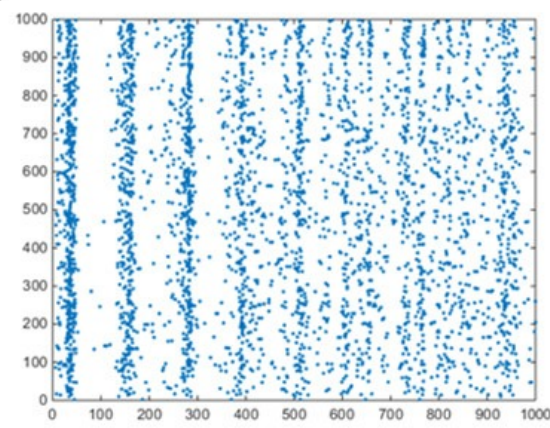

Figure 2. Action potential events for several neural networks. $\mathrm{X}$ axis is time (ms), Y axis represents the 1000 neurons of the network. a) Healthy network, b) Network with $40 \%$ of active neurons, c) Stimulated damaged network at $\mathrm{SF}=2.5$. 
The response of the previously described neural network is present in Figure 2. Figure 2a shows the temporal appearance of the activation of action potentials in a healthy network. A periodicity of the action potentials can be clearly observed. If neurodegenerative pathology is present that makes just $40 \%$ of neurons remain active, the results are the ones presented in Figure 2b. In this second case the periodicity of action potentials is almost lost, as it can be appreciated in the figure. If optical stimulation is switched on over the optical network as a stimulation factor (SF), Figure $2 \mathrm{c}$ is obtained. In this last case the synchronicity of the action potentials is recovered, as it can be easily appreciated.

Another very relevant issue is the analysis of the frequency bands $(\delta, \theta, \alpha, \beta$ and $\gamma)$ of the action potentials events. This analysis can be made for neural networks with different amounts of active neurons, under several optical stimulation conditions. The results appear in Figure 3.

a)

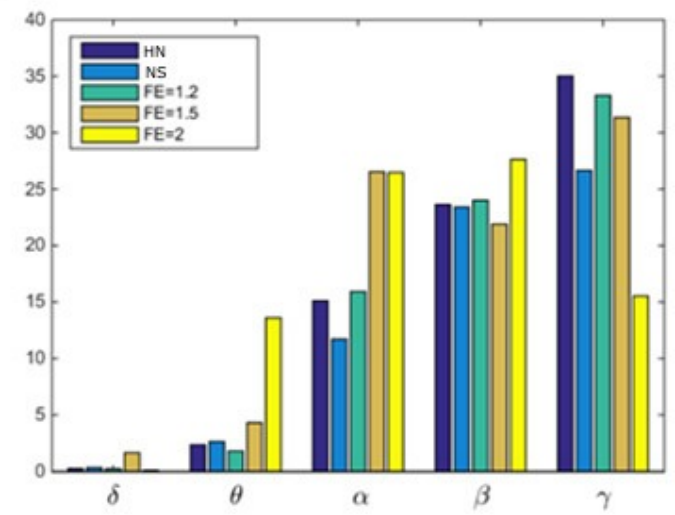

b)

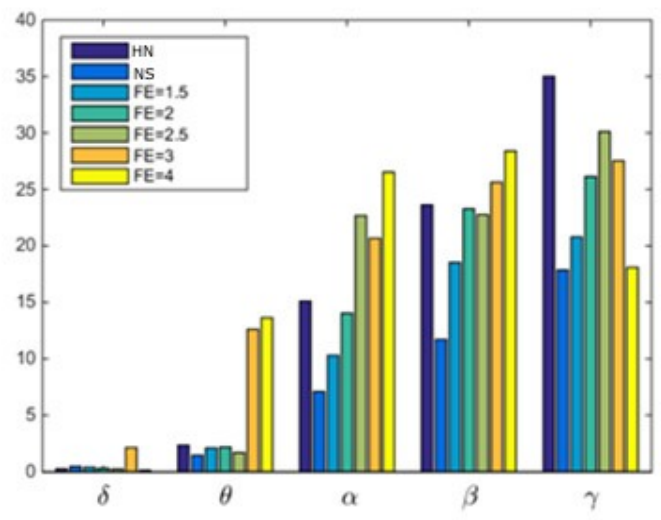

Figure 3. Spectral power as a percentage over the total spectrum energy (Y axis) for the different frequency bands for a healthy network (HN), a damaged network with no stimulation (NS), and several optical stimulation values for FE (equal to ST). a) Damaged network with $80 \%$ of active neurons, b) Damaged network with $40 \%$ of active neurons.

According to Figure 3, optical stimulation modified the frequency power at the different frequency bands. In this sense, it is possible to try to approach the behavior of a damaged network to that of a healthy network. This recovery is easier with $80 \%$ of active neurons (Figure 3a), when compared with only $40 \%$ of active neurons (Figure $3 \mathrm{~b}$ ). Apart from that, there is a tendency to accumulate energy at lower frequency bands when the intensity of the optical stimulation increases. From these results, it would be possible to evaluate the particular optical stimulation parameters needed for the recovery of damaged optical networks.

\section{CONCLUSIONS}

Along this work the effects of Optical Neural Stimulation have been analyzed. The analysis was made by a mesh-based Monte Carlo model of optical propagation, combined with a model of neural networks. Optical stimulation was assumed to be Gaussian. The results show the effect of neural damage on the periodicity of action potentials events, and the partal recovery by optical stimulation. This effect is also appreciated in the analysis of the frequency bands of the brain. The obtained results could be used to determine the proper ONS parameters to induce specific changes in brain activity affected by neurodegenerative diseases.

\section{ACKNOWLEDGEMENTS}

This work has been partially supported by the project MAT2012-38664-C02-01 of the Spanish Ministery of Economy and Competitiveness and by San Cándido Foundation. 


\section{REFERENCES}

[1] Booth, Statistical Office of the European Communities, "Population structure and ageing", EUROSTAT, May 2014, <http://epp.eurostat.ec.europa.eu/statistics_explained/index.php/Population_structure_and_ageing > (20 May 2015).

[2] Fanjul-Vélez, F., Salas-García, I., Ortega-Quijano, N., and Arce-Diego, J. L., "FDTD-based Transcranial Magnetic Stimulation model applied to specific neurodegenerative disorders," Comput. Meth. Prog. Bio., 118(1), 34-43 (2015).

[3] Guleyupoglu, B., Schestatsky, P., Edwardsd, D., Fregnic, F. and Bikson, M., "Classification of methods in transcranial Electrical Stimulation (tES) and evolving strategy from historical approaches to contemporary innovations," J. Neurosci. Methods 219(2), 297-311 (2013).

[4] Wells, J., Kao, C., Mariappan, K., Albea, J., Jansen, E. D., Konrad, P., Mahadevan-Jansen, A., “Optical stimulation of neural tissue in vivo," Opt. Lett. 30(5), 504-506 (2005).

[5] Ortega-Quijano, N., Fanjul-Vélez, F., Salas-García, I. and Arce-Diego, J. L., "Numerical Modeling of Optical Radiation Propagation in a Realistic Model of Adult Human Head," Proc. IFMBE 41, 1697-1682 (2013).

[6] Fang Q., "Mesh-based Monte Carlo method using fast ray-tracing in Plücker coordinates," Biomed. Opt. Express, 32(1), 165-175 (2010).

[7] Hodgkin, A. L. and Huxley, A. F., "A Quantitative Description of Membrane Current and Its Application to Conduction and Excitation in Nerve," J. Physiol., 117(4), 500-544 (1952).

[8] Izhikevich, E. M., "Simple Model of Spiking Neurons," IEEE Trans. Neural Netw., 14(6), 1569-1572 (2003).

[9] Wells, J., Kao, C., Konrad, P., Milner, T., Kim, J., Mahadevan-Jansen, A., and Jansen, E. D., "Biophysical Mechanisms of Transient Optical Stimulation of Peripheral Nerve," Biophys. J., 93(7), 2567-2580 (2007). 